\title{
Automatic Centerline Extraction of Irregular Tubular Structures Using Probability Volumes from Multiphoton Imaging
}

\author{
A. Santamaría-Pang ${ }^{1}$, C.M. Colbert ${ }^{2}$, P. Saggau ${ }^{3}$, and I.A. Kakadiaris ${ }^{1}$ \\ ${ }^{1}$ Computational Biomedicine Lab, Dept. of CS, Univ. of Houston, Houston, TX \\ ${ }^{2}$ Dept. of Biology and Biochemistry, Univ. of Houston, Houston, TX \\ ${ }^{3}$ Dept. of Neuroscience, Baylor College of Medicine, Houston, TX
}

\begin{abstract}
In this paper, we present a general framework for extracting $3 \mathrm{D}$ centerlines from volumetric datasets. Unlike the majority of previous approaches, we do not require a prior segmentation of the volume nor we do assume any particular tubular shape. Centerline extraction is performed using a morphology-guided level set model. Our approach consists of: i) learning the structural patterns of a tubular-like object, and ii) estimating the centerline of a tubular object as the path with minimal cost with respect to outward flux in gray level images. Such shortest path is found by solving the Eikonal equation. We compare the performance of our method with existing approaches in synthetic, CT, and multiphoton 3D images, obtaining substantial improvements, especially in the case of irregular tubular objects.
\end{abstract}

\section{Introduction}

A central goal of modern neuroscience is to elucidate the computational principles and cellular mechanisms that underlie brain function in both normal and diseased states. For over a century it has been appreciated that neuronal morphologies are highly variable (presenting tubular-like shapes), and today it is clear that morphology contributes significantly to the unique computations performed by different classes of neurons.

To achieve realistic modeling of neuronal morphology, one must use centerline extraction from imaging data of dendrites - highly irregular tubular structures (Fig. 1). The challenges in centerline extraction of dendritic structures include: i) a poor signal-to-noise ratio, ii) the objects of interest are at the limit of optical imaging (resolution is typically on the order of $0.2 \mu \mathrm{m}$ ), iii) a non-homogeneous distribution of optical intensity throughout the cell, and, most importantly, iv) there is an extreme variation in shape among dendrites.

Different methods have been proposed to extract the medial axis of tubular structures by using the distance transform to identify the centerline. In Deschamps et al. 1] centerline extraction of segmented tubular objects is accomplished by evolving monotonic fronts, where the cost function is a distance function from the edges of the binary object of interest. Similarly, Hossouna et al.

N. Ayache, S. Ourselin, A. Maeder (Eds.): MICCAI 2007, Part II, LNCS 4792, pp. 486 494, 2007.

(C) Springer-Verlag Berlin Heidelberg 2007 


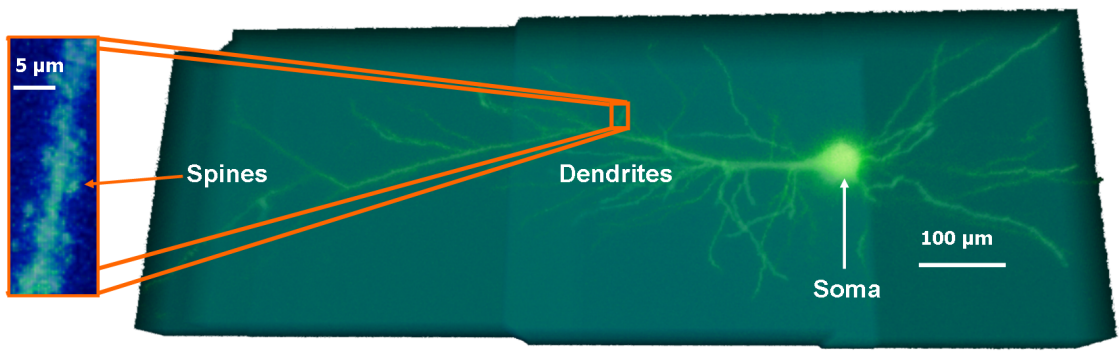

Fig. 1. Neuron morphology

2 proposed centerline extraction from monotonic front evolution, where the centerline follows the maximal distance from the boundary of the binary object. In recent work, Bouix et al. 3] used the average outward flux through a Jordan curve. In this case, the gradient vector field of the Euclidean distance to the boundary was exploited to approximate the centerlines. However, these methods require binary images to make the result dependent on the quality of the segmentation.

In work related to vessel segmentation, Vasilevskiy and Siddiqi [4, and Nain et al. [5] developed a geometric flow framework to perform 2D and 3D vessel segmentation using prior knowledge of the vessel shape. Kimmel [6] offers a review of different geometric measures in variational methods. Complementary, elegant statistical methods for vessel segmentation have been proposed by Florin et al. [7, where shape variations of the vessel are learned on-line during the segmentation. Similarly, in [8] we presented a method for detection of tubular structures by learning different tubular shapes. Such methods allow segmentation or detection of the overall tubular model as opposed to enhancing the centerline of the tubular object.

In this paper, we propose a robust centerline extraction method of tubularlike structures with considerable cross sectional and radius variation. Our main contributions are: i) a novel approach for learning and predicting generalized 3D centerline models, ii) a centerline extraction method using a shortest path formulation in terms of regions of maxima outer flux, and iii) centerline extraction is accomplished without prior segmentation.

\section{Materials and Methods}

Experimental Data: Multiphoton data correspond to cells of interest from mouse cortical CA1 pyramidal neuron cells (Fig. 4 (k) from rat hippocampi. Images were acquired with a customized multiphoton Galvo microscope and loaded with Alexa Fluor 594 dye. We have collected twelve image datasets consisting of seven or more partially overlapping stacks with approximately size of $640 \times 480 \times 150$ each, with voxel size $0.3 \mu \mathrm{m}$ in the $x-y$ axis and $1.0 \mu \mathrm{m}$ in the $z$ axis. Excitation wavelength was set to $810 \mathrm{~nm}$ while the lens and index of refraction both correspond to water. 

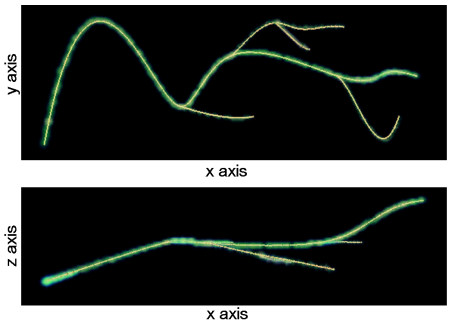

(a)

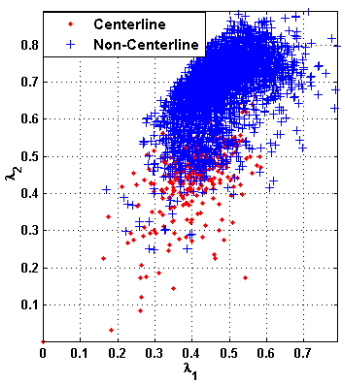

(b)

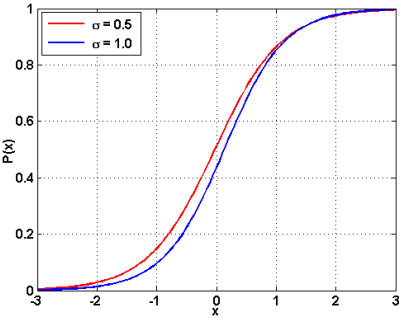

(c)

Fig. 2. (a) Visualization of a three-dimensional tubular model, (b) distribution of the normalized eigenvalues $\lambda_{1}, \lambda_{2}$ (notice the overlap region between the classes), and (c) estimated sigmoid function for different values of $\sigma$

Algorithm Overview: Dendrite centerline extraction is accomplished by the following steps: 1) frames-based denoising, 2) learning generalized tubular models, 3) probabilistic wave propagation, and 4) centerline extraction.

Step 1 - Frames-based Denoising: We constructed a non-separable 3D Parseval frame to remove noise that follows a Poisson distribution from optical images. Our method uses 3D multidirectional filters, which are used both for analysis and synthesis. Noise is suppressed by shrinking frame coefficients to zero according to an ensemble operator 9 .

Step 2 - Learning Generalized Tubular Models: Dendritic neurons do not follow complete cylindrical or elliptical shape patterns as vessels; instead they present highly irregular tubular-like patterns in addition to adjoining structures (Fig. 1). Different types of tubular measures have been proposed in the literature (Frangi et al. [10, Sato et al. [11]) by assuming ideal cylindrical or elliptical geometrical shapes. These measures discriminate among different structural features such as: plates, lines, and blob-like structures. However, they cannot be applied to irregular tubular structures since the structural information they contain does not fulfill the hypothesis of the assumed model.

We hypothesize that regular and irregular cylindrical shape models lead to different probability density functions that can encode tubular shape descriptors. Then, we reformulate the problem of detecting tubular structures to learning a structural tubular model from the object of interest itself (as opposed to defining an ideal tubular measure).

Let us consider the volumetric representation of a 3D tubular structure (Fig. 2) and use the centerline to select the eigenvalues which correspond to the centerline itself. The selected eigenvalues capture shape information along the tubular model, which includes branching points and branches with different diameters at a given scale $\sigma$ 10. Then, we want to define a function which takes high values in the centerline of the object and low values in the boundary. Figure [2(b) depicts the class distribution of the normalized eigenvalues. Note that there is high overlap of the two classes. Thus, in order to estimate the class 
distributions, an algorithm with flexible decision limits and still able to generalize well (due to the sparseness of the data) is required.

Support Vector Machines (SVMs) estimate a decision function $f(\mathbf{x})$ :

$$
f(\mathbf{x})=\sum_{i=1}^{l} y_{i} \alpha_{i} K\left(\mathbf{x}_{i}, \mathbf{x}\right)+b
$$

where $\mathbf{x} \in \mathbb{R}^{n}, \mathbf{x}_{i} \in \mathbb{R}^{n}, i=1, \ldots, l$ are the support vectors, $K$ is a kernel function, $y_{i} \in\{-1,1\}$, with $\alpha_{i}>0$, such that $\sum_{i=1}^{l} y_{i} \alpha_{i}=1$, and $b$ is a learned constant. For classification problems, class prediction is performed by finding a threshold value for the function $f$ and by assigning a penalty value.

Instead of using SVMs for classification, we use them to robustly estimate a posterior probability density function using the approached proposed by Platt [12]. The posterior probability $P(y=1 \mid f)$ is approximated by fitting a parametric sigmoid function as:

$$
P(y=1 \mid f)=\frac{1}{1+e^{(A f(\mathbf{x})+B)}},
$$

where the parameters $A$ and $B$ are computed by defining a new training set $\left(f_{i}, t_{i}\right)$, with $t_{i}=\frac{\left(y_{i}+1\right)}{2}$ and using a maximum likelihood estimation:

$$
\min _{Z=(A, B)} F(Z)=-\sum_{i=1}^{l}\left[t_{i} \log \left(p_{i}\right)+\left(1-t_{i}\right) \log \left(1-p_{i}\right)\right],
$$

where $p_{i}=\frac{1}{1+e^{\left(A f_{i}+B\right)}}$ and $f_{i}=f\left(x_{i}\right)$. Figure 2] $[\mathrm{c})$ depicts the estimated sigmoid function for different values of $\sigma$.

Step 3 - Probabilistic Wave Propagation: Given two points $p_{0}, p \in \mathbb{R}^{3}$, the optimal path between $p_{0}$ and $p$ with respect to a cost function $G(\mathbf{x})$, is defined as the path $c(s)=\{x(s), y(s), z(s)\}$ that minimizes the function $T(p)$ defined as:

$$
T(p)=\min _{c} \int_{p_{0}}^{p} G(c(s)) d s .
$$

where $c(0)=p_{0}, c(L)=p, L$ is the total Euclidian arc-length, and $d s^{2}=d x^{2}+d y^{2}+d z^{2}$.

The level set $C(t)=\{(x, y, z): T(x, y, z)=t\}$ is a strictly monotonic front and it is the set of points that can be reached from $p_{0}$ with minimum cost at time $t$. Assume that $C$ evolves according to:

$$
\frac{\partial C(\mathbf{x})}{\partial t}=\frac{1}{G(\mathbf{x})} \mathcal{N}_{(\mathbf{x})},
$$

where $\mathcal{N}_{(\mathbf{x})}$ is a normal vector to the surface $C$ at the point $\mathbf{x}$. Then $T(C(p, t))=t$ implies the well known Eikonal equation [1] :

$$
U(\mathbf{x})\|\nabla T(\mathbf{x})\|=1, \quad \text { with } T\left(p_{0}\right)=0
$$

where $U>0$ and $U(\mathbf{x})=1 / G(\mathbf{x})$ is the speed of the front propagation. 


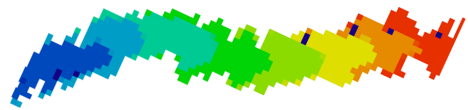

(a)

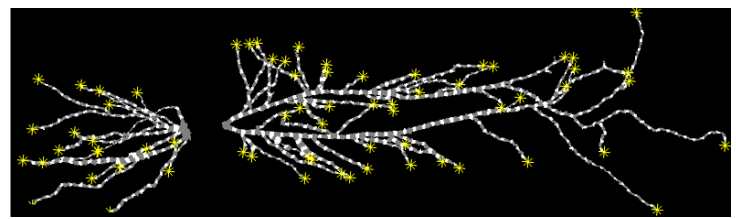

(b)

Fig. 3. (a) Propagation of the front with maximal curvature at the centerline of the dendrite segment. (b) Detection of the terminating points of dendrites.

If we relate Eq. 5 with the inward flux of a given vector field $\mathbf{V}$ defined in $\mathbb{R}^{3}$, as it was proved by Vasilevskiy and Siddiqi [4, the direction of the inward flux of $\mathbf{V}$ through the surface $C$ is increasing most rapidly according to: $\frac{\partial C(\mathbf{x})}{\partial t}=\operatorname{div}(\mathbf{V}(\mathbf{x})) \mathcal{N}_{(\mathbf{x})}$. The divergence of $\mathbf{V}$ can be approximated as:

$$
\operatorname{div}(\mathbf{V}) \equiv \lim _{\Delta V \rightarrow 0} \frac{1}{\Delta V} \iint_{S} \mathbf{V} \cdot \mathbf{n} d S=\frac{\partial \mathbf{V}_{x}}{\partial x}+\frac{\partial \mathbf{V}_{y}}{\partial y}+\frac{\partial \mathbf{V}_{z}}{\partial z}
$$

where $\mathbf{n}$ is the outward unit vector, normal to the surface $S$ surrounding a volume element $\Delta V$. From Eq. 7] it can be deduced that the divergence of $\mathbf{V}$ at a point $\mathbf{x}$ is the flux of $\mathbf{V}$ per unit of volume. If $\operatorname{div}(\mathbf{V}(\mathbf{x}))>0$, we say that $\mathbf{x}$ is a source point and the outward flux is positive, and if $\operatorname{div}(\mathbf{V}(\mathbf{x}))<0$, we say that $\mathbf{x}$ is a sink point and the outward flux is negative [4.

Next, we propose to define an energy function $U$ such that the embedded level set $C$ evolves with higher curvature at the center of a tubular structure, guided by: i) a tubular morphological operator, and ii) the outward flux in gray level images governed by the following partial differential equation:

$$
\left[g(P(\mathbf{x})) \operatorname{div}\left(-\frac{\nabla I(\mathbf{x})}{\|\nabla I(\mathbf{x})\|}\right)\right]\|\nabla T(\mathbf{x})\|=1, \quad \text { with } T\left(p_{0}\right)=0
$$

which is a case of the Eikonal Eq. 6. The term $g(P(\mathbf{x}))$ is a probabilistic morphological operator composed of a non-negative function $g$, and the posterior probability density function $P(y=1 \mid f(\mathbf{x}))$ from a parametric sigmoid function (Eq.2); it is used for enhancement of the centerline of tubular-like objects. It favors maximum propagation of $C$ at the centerlines, preventing the front from propagating outside the tubular object (Fig. $3(\mathrm{a})$. The second term $\operatorname{div}\left(-\frac{\nabla I(\mathbf{x})}{\|\nabla I(\mathbf{x})\|}\right)$ is the mean 3D curvature $k$ of the front $C$ [4], and it favors fast front propagations orthogonal to the gradient $\nabla I(\mathbf{x})$.

Step 4 - Centerline Extraction: For the specific application presented in this paper, morphological reconstruction is performed in four sub-steps. In the first sub-step, the soma center point $p_{0}$ is automatically detected and a front with low curvature from $p_{0}$ is propagated. This is always possible since in Eq. 8 we can set the term $\operatorname{div}\left(-\frac{\nabla I(\mathbf{x})}{\|\nabla I(\mathbf{x})\|}\right)=1$, and $g$ can be a constant function in regions with greater or equal to a given posterior probability value. The surface evolution 
produces a distance map that captures the distance from $p_{0}$ to every voxel. In the second sub-step, terminating voxels are found by creating a number of connected components and finding those components with maximum distance from $p_{0}$. In the third sub-step, a wave starting from the initial voxel $p_{0}$ is initiated according to Eq. 8. Finally, centerlines are extracted by marching along the gradient from the terminating voxels to the initial voxel $p_{0}$ (note that convergence is always guaranteed since the global minimum corresponds to $\left.p_{0}\right)$. Figure 3 (b) depicts the terminating voxels (marked in yellow) and the discretization of the initial distance map (overlapping areas marked in white).

\section{Results}

We have applied our method to both synthetic and real data. We created synthetic data to: i) learn a generic tubular shape model, and ii) detect tubular structures in unseen examples from synthetic and CT data. The model to be learned is depicted in Fig. 2(a) Its morphological properties include: i) variation of intensity, ii) radius variation from 0.5 to $1.5 \mu \mathrm{m}$, iii) variety of branching sections, and iv) high and low curvature segments. Voxel size was isotropic and it was set to $1.0 \mu \mathrm{m}$. The real data are described in Section 2 ,

Training: In both synthetic and real data, parameter selection was performed with a grid search using three-fold cross-validation with different kernels, penalty, and sigma values. With respect to synthetic data, the best performance was obtained using the penalty value equal to 10, a linear kernel, and sigma equal to $0.5 \mu \mathrm{m}$. Optimal SVMs parameters were found to be: $b=4.19$ (Eq. 1) with a number of support vectors equal to 638 , while $A=-1.7955$ and $B=-0.0539$ (Fig. 2) . Concerning multiphoton data, for which the training data consisted of dendrite segments depicted in Fig. Q (g), the best performance was found using a linear kernel and a penalty value equal to 50. The estimated SVMs parameters were: $A=-1.94, B=-0.222$ (Eq. 3), $b=8.269$ (Eq. 1), and $\sigma=1.5 \mu \mathrm{m}$.

Synthetic Data: Figure $4(a)$ depicts an unseen example to detect the centerline. Note that the radius decreases gradually from the bottom $(1.5 \mu \mathrm{m})$ to the top $(0.5 \mu \mathrm{m})$. The centerline is overlaid in white color. Figure $4(\mathrm{~b})$ depicts the predicted centerline with the estimated model in (Fig. 2|(a), while Figure 4(c) depicts the centerline according to Sato's measure [11] $(\sigma=0.5 \mu \mathrm{m}, \alpha=1$, $\beta=1$, and $\gamma=1$ ). Note the difference of these two models, especially at the bottom of the structure. Figure $4(\mathrm{~d})$ depicts the extracted centerline (white line) and the ground truth centerline (brown line). Figures [4](e) (f) illustrate centerline detection from two different CT volumes using the information learned from the synthetic tubular model in Fig. 2)(a). We have created 20 synthetic phantoms with a variety of tubular structures and computed the distance from the extracted centerlines to the ground truth. The maximum and average distances from the extracted centerlines using our method were $0.87 \mu \mathrm{m}$ and $0.57 \mu \mathrm{m}$, while the maximum and average distances from the extracted centerlines using Sato's method were $0.94 \mu \mathrm{m}$ and $0.63 \mu \mathrm{m}$. 


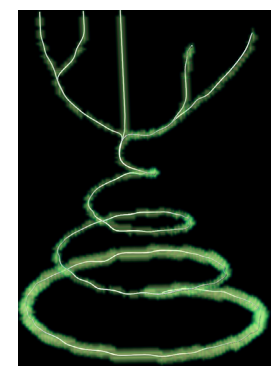

(a)

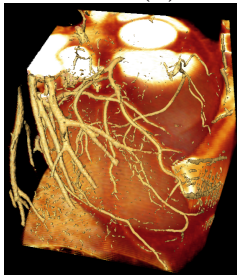

(e)

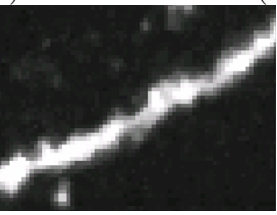

(h)

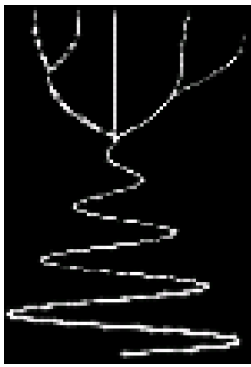

(b)

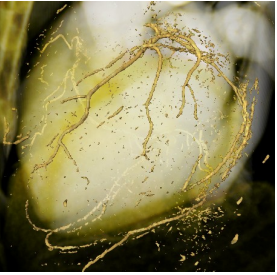

(f)

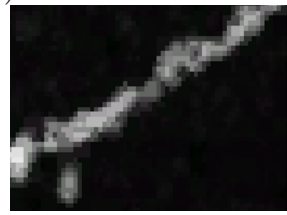

(i)

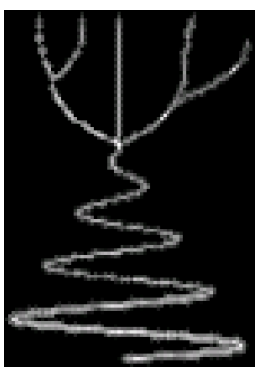

(c)

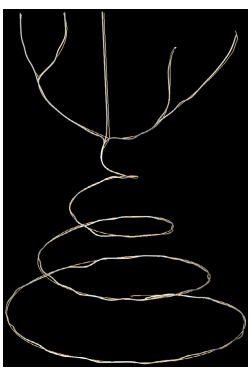

(d)

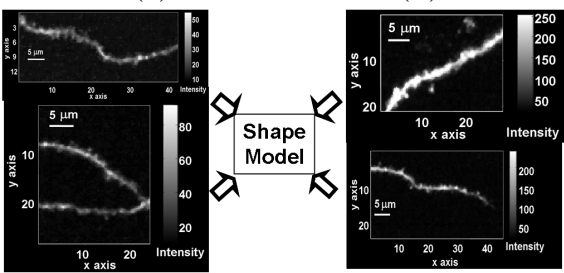

(g)

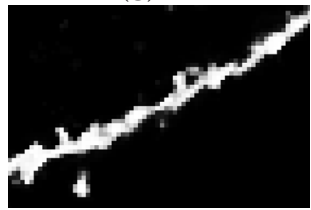

(j)

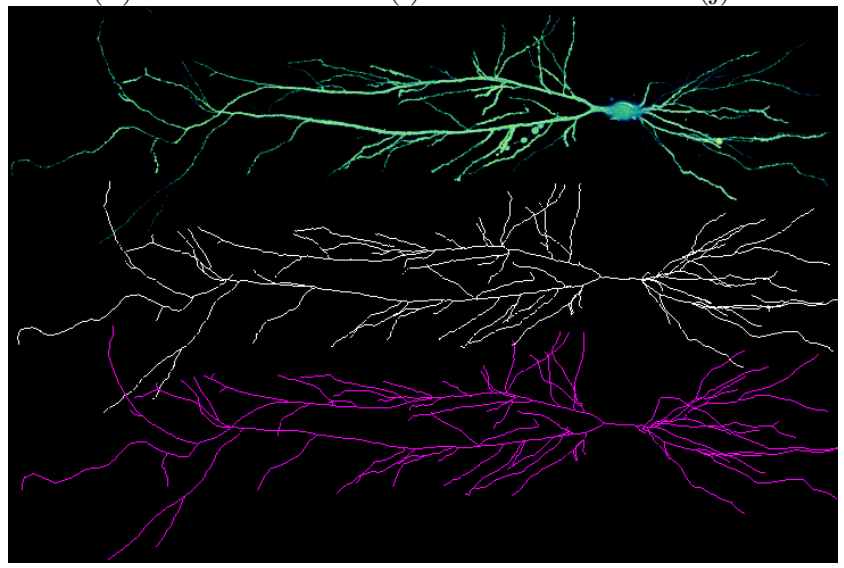

$(\mathrm{k})$

Fig. 4. (a) Visualization of synthetic data with estimated centerline, (b) predicted centerline from tubular model (Fig. 2)(a), (c) predicted centerline from Sato's measure, and (d) extracted centerline (white) and ground true centerline (brown). (e)](f) Tubular structures detected in a CT angiography dataset using the model depicted in Fig. [2(a) (g) Dendrite segments used for training, (h) segment of a given dendrite, (i) after Sato's measure, (j) after our measure. (k) Top: frames-based denoised multiphoton imaging volume, (k) middle: extracted centerlines with our method (white), and (k) bottom: manually extracted centerlines (magenta). 
Multiphoton Microscopy: Figures $4(\mathrm{~h})(\mathrm{j})$ depict a dendrite segment, centerline enhancement using Sato's measure (parameters: $\alpha=1, \beta=1$, and $\gamma=0.5$ ) and our measure respectively. We have performed centerline extraction in all the datasets. In Fig. $4 \mid(\mathrm{k})$ the top subfigure depicts a typical cell with dimensions of 2546 × 912 x 121 voxels, the middle subfigure depicts the extracted centerline (white) with our method, and the bottom subfigure depicts the centerline (magenta) manually traced by an expert. Details about the volume registration and soma detection can be found in [13. When expressing the morphology of a neuron a tree structure, the number of segments that we were able to detect automatically was 149 , compared with 136,150 , and 161 that three human experts were able to trace.

\section{Conclusion}

We have presented a general framework for centerline extraction of tubularlike structures without prior segmentation. Our novel approach consists of: i) learning the structural patterns of a tubular-like structure as opposed to defining a tubularity measure, and ii) combining structural information to be used by a morphology-guided level set. Our method is general since it does not assume any particular tubular shape.

Acknowledgements. We would like to thank B. Losavio and Y. Liang for their valuable assistance. This work was supported in part by NIH 1R01AG027577, NSF IIS-0431144, and NSF IIS-0638875. Any opinions, findings, conclusions or recommendations expressed in this material are the authors' and may not reflect the views of the NIH or NSF.

\section{References}

1. Deschamps, T., Cohen, L.D.: Fast extraction of tubular and tree 3D surfaces with front propagation methods. In: Proc. International Conference on Pattern Recognition, Quebec, Canada, vol. 1, pp. 731-734 (2002)

2. Hassouna, M.S., Farag, A.A., Falk, R.: Differential fly-throughs (DFT): A general framework for computing flight paths. In: Proc. Medical Image Computing and Computer Assisted Intervention, Palm Springs, USA, vol. 1, pp. 654-661 (2005)

3. Bouix, S., Siddiqi, K., Tannenbaum, A.: Flux driven automatic centerline extraction. Medical Image Analysis 9(3), 209-221 (2005)

4. Vasilevskiy, A., Siddiqi, K.: Flux maximizing geometric flows. IEEE Transactions on Pattern Analysis and Machine Intelligence 24(12), 1565-1578 (2002)

5. Nain, D., Yezzi, A.J., Turk, G.: Vessel segmentation using a shape driven flow. In: Proc. Medical Image Computing and Computer Assisted Intervention, Saint-Malo, France, vol. 1, pp. 51-59 (2004)

6. Kimmel, R.: Fast Edge Integration. In: Level Set Methods and their Applications in Computer Vision, Springer, NY (2003) 
7. Florin, C., Paragios, N., Williams, J.: Globally optimal active contours, sequential monte carlo and on-line learning for vessel segmentation. In: Leonardis, A., Bischof, H., Pinz, A. (eds.) ECCV 2006. LNCS, vol. 3953, pp. 476-489. Springer, Heidelberg (2006)

8. Santamaría-Pang, A., Bîldea, T.S., Colbert, C.M., Saggau, P., Kakadiaris, I.A.: Towards segmentation of irregular tubular structures in 3D confocal microscope images. In: Proc. MICCAI International Workshop in Microscopic Image Analysis and Applications in Biology, Copenhangen, Denmark, pp. 78-85 (2006)

9. Santamaría-Pang, A., Bîldea, T.S., Konstantinidis, I., Kakadiaris, I.A.: Adaptive frames-based denoising of confocal microscopy data. In: Proc. Conf. on Acoustics, Speech, and Signal Processing, Toulouse, France, vol. 2, pp. 85-88 (2006)

10. Frangi, A.F., Niessen, W.J., Vincken, K.L., Viergever, M.A.: Multiscale vessel enhancement filtering. In: Proc. Medical Image Computing and Computer Assisted Intervention, Cambridge, USA, vol. 1496, pp. 130-137 (1988)

11. Sato, Y., Nakajima, S., Atsumi, H., Koller, T., Gerig, G., Yoshida, S., Kikinis, R.: 3$\mathrm{D}$ multi-scale line filter for segmentation and visualization of curvilinear structures in medical images. Medical Image Analysis 2(2), 143-168 (1998)

12. Platt, J.: Probabilistic outputs for support vector machines and comparison to regularize likelihood methods. In: Adv. in Large Margin Classifiers, pp. 61-74 (2000)

13. Urban, S., O’Malley, S.M., Walsh, B., Santamaría-Pang, A., Saggau, P., Colbert, C.M., Kakadiaris, I.A.: Automatic reconstruction of dendrite morphology from optical section stacks. In: Proc. ECCV International Workshop on Computer Vision Approaches to Medical Image Analysis, Graz, Austria, pp. 190-201 (2006) 